\title{
A catalog of warps in spiral and lenticular galaxies in the Southern hemisphere
}

\author{
M. L. Sánchez-Saavedra ${ }^{1}$, E. Battaner ${ }^{1}$, A. Guijarro ${ }^{2}$, M. López-Corredoira ${ }^{3}$, and N. Castro-Rodríguez ${ }^{3,4}$ \\ 1 Dpto. Física Teórica y del Cosmos, Universidad de Granada, Avd. Fuentenueva, 18002 Granada, Spain \\ 2 Centro Astronómico Hispano Alemán, 04080 Almería, Spain \\ 3 Astronomisches Institut der Universitat Basel, Venusstrasse 7, Binningen, Switzerland \\ ${ }^{4}$ Instituto de Astrofísica de Canarias, 38205 La Laguna, Tenerife, Spain
}

Received 26 April 2002 / Accepted 22 November 2002

\begin{abstract}
A catalog of optical warps of galaxies is presented. This can be considered complementary to that reported by Sánchez-Saavedra et al. (1990), with 42 galaxies in the northern hemisphere, and to that by Reshetnikov \& Combes (1999), with 60 optical warps. The limits of the present catalog are: $\operatorname{logr} 25>0.60, B_{\mathrm{t}}<14.5, \delta(2000)<0^{\circ},-2.5<t<7$. Therefore, lenticular galaxies have also been considered. This catalog lists 150 warped galaxies out of a sample of 276 edge-on galaxies and covers the whole southern hemisphere, except the Avoidance Zone. It is therefore very suitable for statistical studies of warps. It also provides a source guide for detailed particular observations. We confirm the large frequency of warped spirals: nearly all galaxies are warped. The frequency and warp angle do not present important differences for the different types of spirals. However, no lenticular warped galaxy has been found within the specified limits. This finding constitutes an important restriction for theoretical models.
\end{abstract}

Key words. catalogs - galaxies: structure - galaxies: spiral - galaxies: elliptical and lenticular, cD

\section{Introduction}

As peripheral features, disc warps are better observed in $21 \mathrm{~cm}$. This has been the preferred observational technique since their discovery by Sancisi (1976) and the study by Bosma (1981) until more recent samples such as those analyzed by García-Ruíz (2001) and García-Ruíz et al. (2000). Optical observations provide an important complementary tool. Even if the relation between optical and radio warps remains unclear (García-Ruíz 2001), the great advantage of optical observations lies in the much larger samples available. Catalogs of warped galaxies have been useful to establish observational restrictions to explain warps. Sánchez-Saavedra et al. (1990) first produced a catalog of 42 optical warps in the northern hemisphere out of a sample of 86 galaxies analyzed. The most noticeable result was that, taking into account the probability of non-detection of warps when the line of nodes lies in the plane of the sky, nearly all galaxies are warped, confirming the suggestion made by Bosma (1981) for HI warps. Warps are therefore a universal feature, common for nearly all spiral galaxies. Even this large frequency is a severe restriction for theoretical models. As Reshetnikov and Combes said: "Differential precession wraps any warp", in contrast with the large frequency of real warped galaxies.

Send offprint requests to: M. L. Sánchez-Saavedra, e-mail: malusa@ugr.es
Reshetnikov \& Combes (1999) studied 540 edge-on galaxies, from which a sub-sample of 60 warped galaxies was presented. One of the most noticeable new results reported in their work was that warps were more frequent in denser environments. They also found that the warping mechanism is equally efficient in all types of spirals.

Detailed studies of particular warped galaxies in the optical, such as those by Florido et al. (1991) and by de Grijs (1997), the latter including 44 galaxies, are also of great interest, evidently, but the production of catalogs has greater statistical power.

Here, we have examined all the galaxies contained in LEDA, with $\log 25$ ( $\log 10$ of axis ratio (major/minor axis)) $>0.60, B_{\mathrm{t}}$ (total $B$-magnitude) $<14.5, \delta(2000)<0^{\circ},-2.5<t$ (morphological type code) $<7$. The number of galaxies fulfilling these requirements is 276 , which is our basis for this work, in which the galaxies were analyzed by means of the DSS. Only discs with a warp angle $w a$ (measured from the galactic center to the last observable point with respect to the galactic plane) larger than $4^{\circ}$ are considered to be warped.

To determine the warp angle, $w a$, and other geometrical parameters of a warp, or even the very existence of a warp, subjective criteria were used in previous catalogs and are in turn used in the present one. Objective procedures (Jiménez-Vicente 1998; Jiménez-Vicente et al. 1998) present some problems when applied to a very large number of galaxies and were 
disregarded. More specifically, in some galaxies it was necessary to apply the automatic star removing procedure to an excessively large number of stars, rendering the map highly distorted. Also the separation of real warps and spiral arms was difficult to define, even for galaxies where this separation is clear to the human eye.

Our work presents 150 warps extracted from 276 edge-on galaxies. The recent study by Reshetnikov \& Combes (1998, 1999) extracted 60 warps from 540 edge-on galaxies. It is evident that these authors used stricter criteria to define when a galaxy is definitely warped. Our sample and scope are complementary. The Reshetnikov \& Combes sample is limited by coordinates $0^{\mathrm{h}} \leq \alpha(1950) \leq 14^{\mathrm{h}}, \delta(1950) \leq-17.5^{\circ}$; ours by $\delta(2000) \leq 0^{\circ}$ only. That implies that the study by Reshetnikov $\&$ Combes covers $17 \%$ of the whole sky, whereas ours covers $50 \%$. This, however, is not strictly true because a large part of the Southern Sky is covered by the Zone of Avoidance due to the large extinction near the galactic plane. Taking into account this Avoidance Zone $\left(-15^{\circ} \leq b \leq 15^{\circ}\right)$ our sample would cover about $40 \%$ of the whole sky. Even if based on a smaller number of edge-on galaxies, this large coverage renders the present study more useful for certain statistical tasks, such as the distribution of the orientation of warps.

Another important difference between the work by Reshetnikov \& Combes and the present study is that the former only pays attention to the discs of spiral galaxies while ours includes lenticular galaxies. This inclusion is very important from the theoretical point of view because, roughly speaking, lenticulars have a disc but not gas; in other words, the distribution of stars in lenticulars is very similar to that in spiral galaxies. This is an overgeneralisation, because even if the amount of gas in lenticulars is less than in even late-type spirals, lenticulars do process some gas in the outer parts. Another important difference between lenticulars and spirals is that the former have a dominant bulge. This fact makes analysis less simple, as huge bulges could in principle affect the formation of warps. In any case observations of warps in lenticulars have yet to be made and may introduce decisive restrictions on the mechanisms behind warps.

A large number of theories can be found in the literature: non-spherical dark halos misaligned with the disc (Sparke \& Casertano 1988; Dubinski \& Kuijken 1995), gas infall into the dark matter halo (Ostriker \& Binney 1989; Binney 1992) or directly into the disc (López-Corredoira et al. 2002), interactions with companions or small satellites (Weinberg 1998), intergalactic magnetic fields (Battaner et al. 1990), etc. Kuijken \& García-Ruíz (2001) recently presented a concise review summarizing several mechanisms proposed to explain warps.

The large fraction of warped galaxies seems to exclude one of the most obvious models, based on interactions, but this hypothesis has been reconsidered by Weinberg (1998), assuming a strong tidal amplification by the gravitational wake of a satellite, although this fact was not confirmed by García-Ruíz (2001) who also argued that the warp of the Milky Way induced by the Magellanic Clouds should have been predicted to have a very different orientation from that observed. Warps are common in merging systems (Schwarzkopf \& Dettmar 2001) but it remains unclear whether mergers or interactions can explain all, or at least most, warps.

Warps are more frequent in denser environments (Reshetnikov \& Combes 1999). Early $z \approx 1$ warps were considered by Reshetnikov et al. (2002). Early warps were larger, which favors the interaction model. Other models cannot be completely excluded from the observation of early $z \approx 1$ warps. Magnetic fields were also much stronger and the rate of infall of matter onto a galaxy was higher.

García-Ruíz (2001) observed that, even if galaxies with extended discs may be warped, extended discs are less frequent in denser environments.

The observational study of warps in lenticular galaxies is crucial. If warps are absent or are less common in lenticular galaxies which are gas-poor, those models based on gravitation alone would have difficulty in explaining this fact. Models in which a permanent torque acts on the gas of the galaxy would have the preference. For instance, models like that by Kahn \& Woltjer (1959) or its more recent version by López-Corredoira et al. (2002) would be favored. Note that neither model requires the assumption that galaxies have a large dark matter halo. The magnetic model, in which intergalactic magnetic fields maintain the warp structure, would acquire additional support from this fact.

It therefore seems that the compilation of large samples of warped galaxies, even if they contain just a few parameters about their position, the parent galaxy and the magnitude and shape of the warping, contributes as much as the detailed examination of the HI maps of a few galaxies.

\section{The new catalog}

The catalog presented here is shown in Table 1 . Column 1 gives the pgc number, Col. 2 the galaxy-name or the alternate name, Cols. 3 and 4 the source position for the epoch J2000, the right ascension (al2000, hours decimal value) and declination (de2000, degrees decimal value), Col. 5 the log 10 of apparent diameter (d25 in $0.1^{\prime}$ ), Col. 6 the $\log 10$ of the axis ratio (major axis/minor axis) and Col. 7 the morphological type code $(t)$, (directly adopted from LEDA). Columns 8-11 are the result of our analysis, with the following meaning:

Column 8, labelled $N / S$, specifies the apparent warp rotation, $S$ clockwise, $N$ counterclockwise. The $N$ and $S$ shapes are really two sides of the same galaxy. This difference is therefore completely unimportant from the physical point of view. However, we have kept the information because it is needed when studying the distribution of warps in space, for instance, when considering the orientation of warps in a cluster of galaxies (see for instance, Battaner et al. 1991) $L$ means that only one of the two sides of the galaxy is warped. $U$ means that the two warps are not antisymmetric, i.e. that the apparent warp rotation on the two sides of the edge-on galaxies has opposite directions. In this column, “-” means unwarped and "?" unclear.

Column 9, labelled ( $w a) E-W$, gives the warp angle, defined as the angle between the outermost detected point and the mean position of the plane of symmetry, as defined by the internal unwarped region. $E$ indicates the side of the galaxy closer to the 
Table 1. Sample of spiral galaxies. In the 8th column "-" means unwarped and "?" unclear; in the 9th column "b" means arms, "c" means corrugated and "0" means unwarped.

\begin{tabular}{|c|c|c|c|c|c|c|c|c|c|c|}
\hline pgc & galaxy-name & al2000 & de2000 & $\log d 25$ & $\log 25$ & $t$ & $N / S$ & $(w a) \mathrm{E}-\mathrm{W}\left({ }^{\circ}\right)$ & $\beta\left(^{\circ}\right)$ & $\alpha_{\mathrm{s}}$ \\
\hline 43 & ESO 293-27 & 0.00817 & -40.48400 & 1.32 & 0.60 & 3.9 & $L S$ & $?$ & - & - \\
\hline 474 & MCG-7-1-9 & 0.10585 & -41.48350 & 1.53 & 0.62 & 6.0 & $N$ & $7-7$ & $30-31$ & 0 \\
\hline 627 & NGC 7 & 0.13899 & -29.91700 & 1.39 & 0.67 & 4.9 & $U$ & $7-7$ & $31-31$ & 0 \\
\hline 725 & ESO 241-21 & 0.17223 & -46.41940 & 1.34 & 0.62 & 3.1 & $N$ & $11-10$ & $39-24$ & 0.05 \\
\hline 1335 & ESO 78-22 & 0.34918 & -63.85740 & 1.34 & 0.79 & 4.3 & $S$ & $6-9$ & $17-31$ & 0.2 \\
\hline 1851 & NGC 134 & 0.50616 & -33.24550 & 1.92 & 0.64 & 4.0 & $N$ & $11-8$ & $22-31$ & 0.16 \\
\hline 1942 & ESO 473-25 & 0.53050 & -26.72010 & 1.41 & 0.84 & 4.9 & $N$ & $5-7$ & - & 0.17 \\
\hline 1952 & ESO 79-3 & 0.53390 & -64.25240 & 1.45 & 0.72 & 3.1 & $N$ & $9-7$ & $27-21$ & 0.13 \\
\hline 2228 & NGC 172 & 0.62067 & -22.58500 & 1.31 & 0.72 & 4.0 & $S$ & $7-7$ & - & 0 \\
\hline 2482 & NGC 217 & 0.69267 & -10.02120 & 1.45 & 0.65 & 0.5 & $N$ & $9-10$ & - & 0.05 \\
\hline 2789 & NGC 253 & 0.79252 & -25.28840 & 2.43 & 0.66 & 5.1 & $N$ & $7-9$ & $22-27$ & 0.13 \\
\hline 2800 & MCG-2-3-15 & 0.79624 & -9.83460 & 1.24 & 0.64 & 3.1 & $U$ & $7-7$ & - & 0 \\
\hline 2805 & MCG-2-3-16 & 0.79641 & -9.89970 & 1.48 & 0.82 & 6.7 & $S$ & $14-10$ & - & 0.17 \\
\hline 2820 & NGC 259 & 0.80091 & -2.77630 & 1.46 & 0.65 & 4.0 & $S$ & $7-9$ & $45-45$ & 0.13 \\
\hline 3743 & NGC 360 & 1.04763 & -65.60990 & 1.57 & 0.89 & 4.3 & $N$ & $8-7$ & $22-17$ & 0.07 \\
\hline 4440 & IC 1657 & 1.23536 & -32.65060 & 1.41 & 0.65 & 3.8 & $S$ & $7-11$ & $21-39$ & 0.22 \\
\hline 4912 & ESO 476-5 & 1.35492 & -22.80050 & 1.29 & 0.61 & 3.9 & $N$ & $10-14$ & $27-34$ & 0.17 \\
\hline 5128 & NGC 527 & 1.39947 & -35.11520 & 1.23 & 0.62 & 0.1 & $N$ & $7-7$ & $22-22$ & 0 \\
\hline 5688 & NGC 585 & 1.52839 & -0.93300 & 1.34 & 0.66 & 1.0 & $N$ & $5-3$ & - & 0.25 \\
\hline 6044 & ESO 297-16 & 1.63329 & -40.06770 & 1.20 & 0.61 & 5.9 & $N$ & $9-7$ & $17-16$ & 0.13 \\
\hline 6161 & ESO 413-16 & 1.66594 & -28.69710 & 1.29 & 0.67 & 3.9 & $S$ & $8-7$ & $17-16$ & 0.07 \\
\hline 6242 & ESO 3-4 & 1.69266 & -83.21210 & 1.28 & 0.70 & 4.1 & $S$ & $7-7$ & $22-27$ & 0 \\
\hline 6966 & MCG-1-5-47 & 1.88028 & -3.44720 & 1.48 & 0.87 & 4.8 & $S$ & $2-7$ & - & 0.56 \\
\hline 7298 & ESO 245-10 & 1.94570 & -43.97350 & 1.34 & 0.61 & 3.2 & $?$ & $?$ & - & - \\
\hline 7306 & IC 176 & 1.94814 & -2.01910 & 1.29 & 0.68 & 5.1 & $N$ & $7-7$ & $22-22$ & 0 \\
\hline 7427 & ESO 297-37 & 1.97033 & -39.54460 & 1.27 & 0.67 & 4.1 & $N$ & $3-7$ & - & 0.4 \\
\hline 8326 & ESO 30-9 & 2.17801 & -75.03880 & 1.40 & 0.61 & 4.8 & $S$ & $4-5$ & - & 0.11 \\
\hline 8581 & MCG-1-6-77 & 2.24055 & -7.36840 & 1.36 & 0.78 & 2.7 & $?$ & $?$ & - & - \\
\hline 8673 & IC 217 & 2.26957 & -11.92660 & 1.34 & 0.67 & 5.8 & $U$ & $5-6$ & - & 0.09 \\
\hline 9582 & NGC 964 & 2.51835 & -36.03420 & 1.33 & 0.60 & 1.9 & $S$ & $3-6$ & - & 0.33 \\
\hline 10645 & ESO 546-25 & 2.81346 & -19.97110 & 1.16 & 0.73 & 3.6 & - & $0-0$ & - & - \\
\hline 10965 & NGC 1145 & 2.90931 & -18.63550 & 1.47 & 0.74 & 5.1 & $N$ & $?-4$ & - & - \\
\hline 11198 & MCG-2-8-33 & 2.96379 & -10.16720 & 1.45 & 0.63 & 0.2 & $N$ & $14-11$ & $22-16$ & 0.12 \\
\hline 11595 & ESO 248-2 & 3.08523 & -45.96350 & 1.47 & 0.69 & 6.9 & $S$ & $10-10$ & $18-18$ & 0 \\
\hline 11659 & NGC 1244 & 3.10866 & -66.77640 & 1.26 & 0.63 & 2.1 & $S$ & $6-6$ & 9-9 & 0 \\
\hline 11851 & IC 1898 & 3.17224 & -22.40440 & 1.53 & 0.77 & 5.8 & $N$ & $5-3$ & $23-11$ & 0.25 \\
\hline 11931 & NGC 1247 & 3.20399 & -10.48070 & 1.54 & 0.82 & 3.8 & $N$ & $4-5$ & - & 0.11 \\
\hline 12521 & NGC 1301 & 3.34317 & -18.71590 & 1.34 & 0.66 & 4.2 & $N$ & $9-6$ & $17-11$ & 0.2 \\
\hline 13171 & IC 1952 & 3.55728 & -23.71280 & 1.40 & 0.66 & 4.1 & $N$ & $8-6$ & $18-13$ & 0.14 \\
\hline 13458 & NGC 1406 & 3.65626 & -31.32200 & 1.59 & 0.70 & 4.4 & $S$ & $3-5$ & $20-11$ & 0.25 \\
\hline 13569 & NGC 1422 & 3.69191 & -21.68240 & 1.36 & 0.63 & 2.4 & - & $0-0$ & - & - \\
\hline 13620 & NGC 1421 & 3.70818 & -13.48830 & 1.54 & 0.60 & 4.1 & ? & ? & - & - \\
\hline 13646 & MCG-2-10-9 & 3.71559 & -12.91570 & 1.50 & 0.90 & 5.0 & $N$ & $7-5$ & $27-18$ & 0.17 \\
\hline 13727 & NGC 1448 & 3.74221 & -44.64400 & 1.88 & 0.65 & 5.9 & $\mathrm{~S}$ & $9-8$ & $45-25$ & 0.06 \\
\hline 13809 & ESO 358-63 & 3.77189 & -34.94240 & 1.69 & 0.64 & 4.7 & $L S$ & $3-0$ & - & 1 \\
\hline 13912 & IC 2000 & 3.81879 & -48.85810 & 1.63 & 0.74 & 6.1 & $N$ & $5-5$ & - & 0 \\
\hline 13926 & ESO 482-46 & 3.82835 & -26.99350 & 1.55 & 0.82 & 5.1 & ? & $\mathrm{c}$ & - & - \\
\hline 14071 & NGC 1484 & 3.90487 & -36.97110 & 1.40 & 0.69 & 3.5 & $S$ & $5-5$ & - & 0 \\
\hline
\end{tabular}


Table 1. continued.

\begin{tabular}{|c|c|c|c|c|c|c|c|c|c|c|}
\hline pgc & galaxy-name & al2000 & de2000 & $\operatorname{logd} 25$ & $\log 25$ & $\bar{t}$ & $N / S$ & $(w a) \mathrm{E}-\mathrm{W}\left(\left(^{\circ}\right)\right.$ & $\beta\left(^{\circ}\right)$ & $\overline{\alpha_{\mathrm{s}}}$ \\
\hline 14190 & NGC 1495 & 3.97255 & -44.46650 & 1.46 & 0.73 & 5.1 & $S$ & $4-9$ & - & 0.38 \\
\hline 14255 & NGC 1511A & 4.00542 & -67.80730 & 1.28 & 0.63 & 1.3 & $S$ & $7-7$ & - & 0 \\
\hline 14259 & ESO 483-6 & 4.00724 & -25.18150 & 1.43 & 0.79 & 3.2 & $S$ & $5-7$ & - & 0.17 \\
\hline 14337 & ESO 117-19 & 4.04233 & -62.31570 & 1.31 & 0.76 & 3.9 & - & $0-0$ & - & - \\
\hline 14397 & NGC 1515 & 4.06748 & -54.10270 & 1.72 & 0.62 & 4.0 & $N$ & $5-8$ & $10-10$ & 0.23 \\
\hline 14824 & IC 2058 & 4.29847 & -55.93440 & 1.49 & 0.94 & 6.6 & $S$ & $4-6$ & - & 0.2 \\
\hline 15455 & ESO 202-35 & 4.53769 & -49.67520 & 1.44 & 0.81 & 3.3 & $N$ & $5-5$ & $16-11$ & 0 \\
\hline 15635 & NGC 1622 & 4.61015 & -3.18920 & 1.52 & 0.73 & 2.0 & $S$ & $5-10$ & $13-21$ & 0.33 \\
\hline 15654 & NGC 1625 & 4.61841 & -3.30340 & 1.37 & 0.64 & 3.1 & $S$ & $6-6$ & $11-?$ & 0 \\
\hline 15674 & NGC 1628 & 4.62671 & -4.71480 & 1.27 & 0.64 & 3.1 & $N$ & $8-9$ & $11-17$ & 0.06 \\
\hline 15749 & ESO $157-49$ & 4.66038 & -53.01200 & 1.28 & 0.66 & 4.1 & $S$ & $11-11$ & $21-21$ & 0 \\
\hline 15758 & IC 2103 & 4.66323 & -76.83680 & 1.28 & 0.76 & 4.9 & $?$ & $\mathrm{~b}$ & - & - \\
\hline 16144 & IC 2098 & 4.84561 & -5.41870 & 1.35 & 0.87 & 5.9 & - & $0-0$ & - & - \\
\hline 16168 & MCG-1-13-22 & 4.85739 & -3.12210 & 1.22 & 0.60 & 3.0 & $S$ & $4-4$ & - & 0 \\
\hline 16187 & IC 2101 & 4.86165 & -6.22970 & 1.23 & 0.66 & 5.0 & ? & ? & - & - \\
\hline 16199 & ESO 361-15 & 4.86601 & -33.17860 & 1.44 & 0.68 & 6.1 & $S$ & $7-7$ & $9-17$ & 0 \\
\hline 16239 & NGC 1686 & 4.88184 & -15.34620 & 1.25 & 0.71 & 4.0 & $N$ & $4-7$ & -16 & 0.27 \\
\hline 16636 & MCG-1-13-50 & 5.05475 & -2.93560 & 1.39 & 0.82 & 3.0 & - & $0-0$ & - & - \\
\hline 16849 & NGC 1827 & 5.16768 & -36.95890 & 1.48 & 0.76 & 5.9 & $?$ & - & - & - \\
\hline 16893 & MCG-1-14-3 & 5.19493 & -3.09160 & 1.16 & 0.67 & 3.1 & $\mathrm{~N}$ & $7-5$ & - & 0.17 \\
\hline 17027 & ESO 362-11 & 5.27748 & -37.10210 & 1.68 & 0.75 & 4.1 & $N$ & $6-4$ & $11-6$ & 0.2 \\
\hline 17056 & IC 407 & 5.29517 & -15.52370 & 1.24 & 0.70 & 4.9 & $S$ & $6-6$ & - & 0 \\
\hline 17174 & NGC 1886 & 5.36352 & -23.81260 & 1.51 & 0.77 & 3.9 & - & $0-0$ & - & - \\
\hline 17248 & MCG-2-14-16 & 5.41515 & -12.68870 & 1.28 & 0.77 & 2.0 & - & $0-0$ & - & - \\
\hline 17433 & NGC 1963 & 5.55355 & -36.39980 & 1.46 & 0.78 & 5.8 & $?$ & $?$ & - & - \\
\hline 17969 & ESO 555-2 & 5.84077 & -19.72620 & 1.34 & 0.74 & 3.9 & $S$ & $6-6$ & - & 0 \\
\hline 17993 & ESO 160-2 & 5.85418 & -53.57480 & 1.26 & 0.63 & 3.0 & $?$ & $?$ & - & - \\
\hline 18394 & ESO 5-4 & 6.09456 & -86.63220 & 1.58 & 0.68 & 2.9 & - & $0-0$ & - & - \\
\hline 18437 & ESO 121-6 & 6.12505 & -61.80710 & 1.60 & 0.75 & 5.1 & $S$ & $3-3$ & - & 0 \\
\hline 18765 & ESO 489-29 & 6.28479 & -27.38620 & 1.52 & 0.72 & 3.9 & - & $0-0$ & - & - \\
\hline 18833 & NGC 2221 & 6.33750 & -57.57740 & 1.33 & 0.66 & 1.1 & $U$ & $7-6$ & $18-11$ & 0.08 \\
\hline 19996 & ESO 491-15 & 7.01183 & -27.36820 & 1.35 & 0.68 & 5.0 & $N$ & $3-3$ & - & 0 \\
\hline 20903 & ESO 428-28 & 7.39406 & -30.05070 & 1.39 & 0.84 & 5.3 & $U$ & - & - & - \\
\hline 21338 & ESO $257-19$ & 7.58538 & -46.92470 & 1.40 & 0.74 & 6.2 & $?$ & $b$ & - & - \\
\hline 21815 & ESO $311-12$ & 7.79281 & -41.45170 & 1.57 & 0.74 & 0.1 & $S$ & $3-3$ & $6-8$ & 0 \\
\hline 21822 & ESO 560-13 & 7.79774 & -18.74840 & 1.48 & 0.77 & 4.0 & $N$ & $10-4$ & $18-8$ & 0.4 \\
\hline 22174 & ESO 35-18 & 7.91809 & -76.41310 & 1.53 & 0.65 & 4.9 & - & $0-0$ & - & - \\
\hline 22272 & ESO 494-7 & 7.94852 & -24.90810 & 1.45 & 0.63 & 4.3 & - & $0-0$ & - & - \\
\hline 22338 & ESO 209-9 & 7.97080 & -49.85160 & 1.80 & 0.83 & 6.0 & $N$ & $5-5$ & $9-17$ & 0 \\
\hline 22910 & ESO 89-12 & 8.16682 & -64.93620 & 1.41 & 0.86 & 4.1 & $N$ & $7-7$ & $15-17$ & 0 \\
\hline 23558 & ESO 495-12 & 8.39769 & -25.83820 & 1.27 & 0.67 & 3.0 & $S$ & $11-11$ & $14-17$ & 0 \\
\hline 23672 & IC 2375 & 8.43881 & -13.30300 & 1.28 & 0.70 & 3.0 & $S$ & $17-14$ & $40-27$ & 0.1 \\
\hline 23992 & ESO 562-14 & 8.55493 & -17.95650 & 1.15 & 0.64 & 3.0 & $N$ & $9-10$ & $34-45$ & 0.05 \\
\hline 23997 & NGC 2613 & 8.55626 & -22.97330 & 1.85 & 0.62 & 3.2 & $?$ & $\mathrm{c}$ & - & - \\
\hline 24225 & ESO 563-3 & 8.62196 & -20.93980 & 1.21 & 0.65 & 0.4 & - & $0-0$ & - & - \\
\hline 24479 & ESO 563-14 & 8.71596 & -20.05070 & 1.38 & 0.66 & 6.5 & $?$ & $\mathrm{c}$ & - & - \\
\hline 24685 & ESO 563-21 & 8.78801 & -20.03590 & 1.48 & 0.81 & 4.1 & $S$ & $3-5$ & - & 0.25 \\
\hline 25400 & ESO 60-24 & 9.04451 & -68.22650 & 1.46 & 0.78 & 2.9 & $S$ & $2-5$ & - & 0.43 \\
\hline
\end{tabular}


Table 1. continued.

\begin{tabular}{|c|c|c|c|c|c|c|c|c|c|c|}
\hline pgc & galaxy-name & al2000 & de2000 & $\log d 25$ & $\log 25$ & $\bar{t}$ & $\overline{N / S}$ & $(w a) \mathrm{E}-\mathrm{W}\left({ }^{\circ}\right)$ & $\beta\left(^{\circ}\right)$ & $\overline{\alpha \alpha_{\mathrm{s}}}$ \\
\hline 25886 & MCG-1-24-1 & 9.18087 & -8.88820 & 1.63 & 0.63 & 3.3 & $\mathrm{~S}$ & $5-5$ & - & 0 \\
\hline 25926 & ESO 564-27 & 9.19844 & -20.11760 & 1.65 & 0.94 & 6.1 & $S$ & $2-2$ & - & 0 \\
\hline 26561 & IC 2469 & 9.38368 & -32.45070 & 1.75 & 0.68 & 1.9 & $N$ & $6-6$ & $11-15$ & 0 \\
\hline 26632 & ESO 433-19 & 9.40089 & -28.17720 & 1.15 & 0.76 & 0.8 & - & $0-0$ & - & - \\
\hline 27135 & ESO $373-8$ & 9.55577 & -33.03250 & 1.76 & 0.81 & 6.0 & - & $0-0$ & - & - \\
\hline 27468 & ESO $373-13$ & 9.63908 & -33.86110 & 1.13 & 0.81 & 0.2 & $U$ & $4-4$ & $8-8$ & 0 \\
\hline 27735 & MCG-1-25-22 & 9.70339 & -4.71400 & 1.28 & 0.66 & 4.0 & $N$ & $7-7$ & $16-16$ & 0 \\
\hline 27982 & NGC 2992 & 9.76169 & -14.32750 & 1.56 & 0.62 & 1.0 & $?$ & $?$ & - & - \\
\hline 28117 & ESO 499-5 & 9.78709 & -24.84040 & 1.41 & 0.64 & 5.0 & $S$ & $10-15$ & $31-17$ & 0.2 \\
\hline 28246 & IC 2511 & 9.82372 & -32.84200 & 1.50 & 0.65 & 1.5 & $N$ & $8-10$ & $18-22$ & 0.1 \\
\hline 28283 & IC 2513 & 9.83403 & -32.88580 & 1.49 & 0.60 & 2.2 & - & $0-0$ & - & - \\
\hline 28308 & MCG-2-25-20 & 9.83718 & -12.05720 & 1.25 & 0.78 & 6.8 & $L S$ & $7-0$ & $18-$ & 1 \\
\hline 28778 & ESO 435-14 & 9.96341 & -28.50670 & 1.40 & 0.86 & 4.9 & $N$ & $5-5$ & $11-14$ & 0 \\
\hline 28840 & ESO 435-19 & 9.98502 & -30.24980 & 1.53 & 0.86 & 4.7 & $U$ & $4-5$ & $8-8$ & 0.11 \\
\hline 28909 & IC 2531 & 9.99880 & -29.61540 & 1.83 & 0.96 & 5.0 & - & $0-0$ & - & - \\
\hline 29096 & ESO 316-18 & 10.04541 & -42.09030 & 1.40 & 0.85 & 4.9 & $S$ & $5-6$ & - & 0.09 \\
\hline 29691 & NGC 3157 & 10.19515 & -31.64220 & 1.35 & 0.62 & 5.0 & - & $0-0$ & - & - \\
\hline 29716 & ESO 263-15 & 10.20551 & -47.29430 & 1.49 & 0.91 & 5.8 & - & $0-0$ & - & - \\
\hline 29743 & ESO 436-1 & 10.21327 & -27.83950 & 1.50 & 0.80 & 4.3 & $N$ & $5-5$ & - & 0 \\
\hline 29841 & ESO 567-26 & 10.23436 & -21.97680 & 1.32 & 0.72 & 4.1 & - & $0-0$ & - & - \\
\hline 30716 & ESO 375-26 & 10.45064 & -36.22700 & 1.30 & 0.74 & 4.1 & - & $0-0$ & - & - \\
\hline 30887 & NGC 3263 & 10.48691 & -44.12300 & 1.78 & 0.64 & 5.9 & ? & $?$ & - & - \\
\hline 31154 & ESO 436-34 & 10.54559 & -28.61290 & 1.35 & 0.65 & 3.0 & - & $0-0$ & - & - \\
\hline 31426 & IC 624 & 10.60427 & -8.33410 & 1.41 & 0.61 & 1.2 & $S$ & $6-3$ & $11-11$ & 0.33 \\
\hline 31626 & ESO 437-22 & 10.63830 & -28.88600 & 1.22 & 0.63 & 4.0 & $?$ & c & - & - \\
\hline 31677 & ESO 437-30 & 10.65422 & -30.29910 & 1.50 & 0.73 & 4.0 & $L N$ & $6-0$ & 9- & 1 \\
\hline 31723 & NGC 3333 & 10.66387 & -36.03610 & 1.32 & 0.63 & 4.3 & $L N$ & $6-0$ & 14 & 1 \\
\hline 31919 & ESO 501-80 & 10.71052 & -23.93550 & 1.36 & 0.67 & 5.0 & - & $0-0$ & - & - \\
\hline 31995 & ESO $318-4$ & 10.73068 & -38.26280 & 1.45 & 0.63 & 5.1 & $N$ & $5-5$ & $18-18$ & 0 \\
\hline 32271 & NGC 3390 & 10.80110 & -31.53260 & 1.54 & 0.73 & 2.7 & $N$ & $3-4$ & - & 0.14 \\
\hline 32328 & ESO 264-43 & 10.81209 & -45.42020 & 1.30 & 0.64 & 3.1 & - & $0-0$ & - & - \\
\hline 32550 & ESO 569-14 & 10.85682 & -19.88890 & 1.54 & 0.72 & 6.3 & $L N$ & $8-0$ & $14-$ & 1 \\
\hline 35539 & NGC 3717 & 11.52557 & -30.30770 & 1.78 & 0.64 & 3.1 & $?$ & $?$ & - & - \\
\hline 35861 & NGC 3749 & 11.59794 & -37.99470 & 1.53 & 0.60 & 1.0 & $U$ & $14-14$ & $27-27$ & 0 \\
\hline 36315 & ESO 571-16 & 11.70260 & -18.16960 & 1.21 & 0.66 & 3.9 & $N$ & $6-3$ & $11-8$ & 0.33 \\
\hline 37178 & NGC 3936 & 11.87235 & -26.90650 & 1.60 & 0.73 & 4.4 & - & $0-0$ & - & - \\
\hline 37243 & ESO 379-6 & 11.88444 & -36.63820 & 1.42 & 0.90 & 4.9 & $N$ & $3-2$ & - & 0.2 \\
\hline 37271 & ESO 440-27 & 11.88987 & -28.55320 & 1.64 & 0.83 & 6.7 & $S$ & $4-3$ & $11-11$ & 0.14 \\
\hline 37304 & IC 2974 & 11.89692 & -5.16780 & 1.36 & 0.69 & 4.7 & $S$ & $5-5$ & 9-9 & 0 \\
\hline 37334 & ESO 320-31 & 11.90167 & -39.86450 & 1.42 & 0.88 & 5.1 & - & $0-0$ & - & - \\
\hline 38426 & MCG-2-31-17 & 12.11402 & -11.09900 & 1.31 & 0.77 & 6.0 & S & $7-6$ & $16-10$ & 0.08 \\
\hline 38464 & IC 3005 & 12.12049 & -30.02330 & 1.38 & 0.76 & 5.6 & $N$ & $3-5$ & $6-9$ & 0.25 \\
\hline 38841 & ESO 321-10 & 12.19502 & -38.54850 & 1.31 & 0.83 & 0.8 & - & $0-0$ & - & - \\
\hline 40023 & ESO 380-19 & 12.36725 & -35.79230 & 1.50 & 0.76 & 5.8 & - & $0-0$ & - & - \\
\hline 40284 & NGC 4348 & 12.39832 & -3.44330 & 1.49 & 0.70 & 4.1 & $N$ & $10-11$ & $18-21$ & 0.05 \\
\hline 42684 & ESO 268-33 & 12.70824 & -47.55780 & 1.32 & 0.71 & 4.9 & - & $0-0$ & - & - \\
\hline 42747 & UGC 7883 & 12.71593 & -1.22940 & 1.42 & 0.61 & 6.0 & $N$ & $7-11$ & $13-27$ & 0.22 \\
\hline 43021 & ESO 507-7 & 12.76168 & -26.24320 & 1.41 & 0.82 & 4.0 & - & $0-0$ & - & - \\
\hline
\end{tabular}


Table 1. continued.

\begin{tabular}{|c|c|c|c|c|c|c|c|c|c|c|}
\hline$\overline{\mathrm{pgc}}$ & galaxy-name & al2000 & $\overline{\mathrm{de} 2000}$ & $\log 225$ & logr25 & $\bar{t}$ & $\bar{N} / \mathrm{S}$ & $(w a) \mathrm{E}-\mathrm{W}\left({ }^{\circ}\right)$ & $\bar{\beta}\left({ }^{\circ}\right)$ & $\overline{\alpha_{\mathrm{s}}}$ \\
\hline 43224 & ESO 507-13 & 12.80150 & -27.57800 & 1.25 & 0.62 & 4.1 & $\bar{N}$ & $6-6$ & $11-11$ & 0 \\
\hline 43313 & IC 3799 & 12.81658 & -14.39910 & 1.40 & 0.88 & 6.7 & - & $0-0$ & - & - \\
\hline 43330 & NGC 4700 & 12.81883 & -11.41060 & 1.47 & 0.75 & 4.9 & $N$ & $8-9$ & $11-18$ & 0.06 \\
\hline 43342 & NGC 4703 & 12.82187 & -9.10850 & 1.39 & 0.69 & 3.1 & - & $0-0$ & - & - \\
\hline 43679 & MCG-1-33-32 & 12.87411 & -9.75390 & 1.39 & 0.92 & 6.7 & - & $0-0$ & - & - \\
\hline 44254 & UGC 8067 & 12.95337 & -1.70690 & 1.28 & 0.66 & 3.5 & - & $0-0$ & - & - \\
\hline 44271 & NGC 4835A & 12.95364 & -46.37780 & 1.43 & 0.62 & 5.8 & - & $0-0$ & - & - \\
\hline 44358 & MCG-1-33-60 & 12.96300 & -9.63360 & 1.51 & 0.94 & 6.7 & - & $0-0$ & - & - \\
\hline 44409 & NGC 4835 & 12.96883 & -46.26320 & 1.67 & 0.69 & 4.0 & $?$ & $\mathrm{~b}$ & - & - \\
\hline 44931 & MCG-1-33-71 & 13.03043 & -8.33620 & 1.45 & 0.85 & 4.9 & $N$ & $8-7$ & $8-9$ & 0.07 \\
\hline 44966 & ESO $381-51$ & 13.03531 & -33.11870 & 1.19 & 0.70 & 2.8 & - & $0-0$ & - & - \\
\hline 45006 & MCG-3-33-28 & 13.04054 & -17.67920 & 1.42 & 0.92 & 4.9 & $N$ & $5-7$ & $11-21$ & 0.17 \\
\hline 45098 & ESO $443-42$ & 13.05827 & -29.82900 & 1.46 & 0.83 & 3.1 & $N$ & $7-5$ & $17-14$ & 0.17 \\
\hline 45127 & MCG-1-33-76 & 13.06291 & -5.13370 & 1.27 & 0.61 & 4.9 & $N$ & $6-6$ & $11-11$ & 0 \\
\hline 45279 & NGC 4945 & 13.09060 & -49.47090 & 2.31 & 0.67 & 6.1 & $N$ & $8-8$ & $18-18$ & 0 \\
\hline 45487 & ESO 508-11 & 13.12910 & -22.85680 & 1.48 & 0.79 & 6.7 & - & $0-0$ & - & - \\
\hline 45911 & ESO 576-11 & 13.21811 & -19.97810 & 1.47 & 0.79 & 5.7 & - & $0-0$ & - & - \\
\hline 45952 & NGC 5022 & 13.22530 & -19.54800 & 1.38 & 0.69 & 3.4 & - & $0-0$ & - & - \\
\hline 46441 & NGC 5073 & 13.32241 & -14.84440 & 1.54 & 0.79 & 5.0 & $S$ & $6-6$ & $17-15$ & 0 \\
\hline 46650 & ESO 40-7 & 13.36095 & -77.53510 & 1.46 & 0.80 & 5.1 & $N$ & $2-9$ & $4-14$ & 0.63 \\
\hline 46768 & IC 4231 & 13.38707 & -26.30050 & 1.25 & 0.65 & 4.3 & - & $0-0$ & - & - \\
\hline 46928 & ESO $382-58$ & 13.42014 & -33.65550 & 1.41 & 0.73 & 3.9 & $?$ & $\mathrm{c}$ & - & - \\
\hline 47345 & ESO 383-5 & 13.48987 & -34.27190 & 1.52 & 0.72 & 3.8 & $N$ & $4-8$ & $11-27$ & 0.33 \\
\hline 47394 & NGC 5170 & 13.49692 & -17.96620 & 1.91 & 0.84 & 4.9 & - & $0-0$ & - & - \\
\hline 47948 & ESO 509-74 & 13.59484 & -24.07400 & 1.40 & 0.72 & 4.7 & $N$ & $5-5$ & $11-11$ & 0 \\
\hline 48359 & ESO 220-28 & 13.67018 & -51.14220 & 1.31 & 0.75 & 4.2 & - & $0-0$ & - & - \\
\hline 49106 & IRAS 13471-4839 & 13.83850 & -48.90490 & 1.25 & 0.72 & 3.6 & $S$ & $25-25$ & $?$ & 0 \\
\hline 49129 & ESO 383-91 & 13.84225 & -37.28920 & 1.41 & 0.81 & 6.7 & $N$ & $5-9$ & $11-17$ & 0.29 \\
\hline 49190 & ESO $384-3$ & 13.85615 & -37.62870 & 1.23 & 0.67 & 3.0 & $S$ & $10-9$ & $22-14$ & 0.05 \\
\hline 49586 & NGC 5365A & 13.94436 & -44.00730 & 1.45 & 0.68 & 3.0 & $N$ & $5-5$ & $11-8$ & 0 \\
\hline 49676 & IC 4351 & 13.96504 & -29.31490 & 1.76 & 0.69 & 3.2 & $N$ & $6-7$ & $11-14$ & 0.08 \\
\hline 49750 & NGC 5365B & 13.97766 & -43.96420 & 1.21 & 0.65 & 2.0 & - & $0-0$ & - & - \\
\hline 49788 & ESO 325-42 & 13.98897 & -40.06910 & 1.21 & 0.63 & 3.2 & $S$ & $5-5$ & - & 0 \\
\hline 49836 & ESO 221-22 & 14.00347 & -48.26770 & 1.37 & 0.69 & 6.8 & $S$ & $2-6$ & $4-8$ & 0.5 \\
\hline 50676 & NGC 5496 & 14.19388 & -1.15890 & 1.64 & 0.69 & 6.5 & S & $8-9$ & $39-45$ & 0.06 \\
\hline 50798 & ESO 271-22 & 14.22487 & -45.41360 & 1.41 & 0.73 & 5.9 & - & $0-0$ & - & - \\
\hline 51288 & IC 4402 & 14.35379 & -46.29830 & 1.68 & 0.69 & 3.2 & $?$ & $\mathrm{c}$ & $17-11$ & - \\
\hline 51613 & ESO 1-6 & 14.45741 & -87.77160 & 1.48 & 0.64 & 5.9 & - & $0-0$ & - & - \\
\hline 52410 & IC 4472 & 14.66980 & -44.31610 & 1.35 & 0.66 & 5.0 & - & $0-0$ & - & - \\
\hline 52411 & ESO 512-12 & 14.66983 & -25.77610 & 1.45 & 0.82 & 3.2 & $N$ & $5-5$ & $16-16$ & 0 \\
\hline 52824 & ESO 580-29 & 14.79267 & -19.76510 & 1.34 & 0.80 & 4.9 & $N$ & $6-6$ & $11-11$ & 0 \\
\hline 52991 & ESO 580-41 & 14.84346 & -18.15090 & 1.30 & 0.70 & 4.3 & - & $0-0$ & - & - \\
\hline 53361 & ESO 327-31 & 14.92637 & -38.27700 & 1.36 & 0.74 & 5.0 & - & $0-0$ & - & - \\
\hline 53471 & MCG-7-31-3 & 14.96256 & -43.13190 & 1.18 & 0.70 & 4.4 & $?$ & $?$ & - & - \\
\hline 54348 & ESO 581-25 & 15.22503 & -20.67680 & 1.54 & 0.70 & 6.9 & $?$ & $\mathrm{c}$ & - & - \\
\hline 54392 & ESO 274-1 & 15.23711 & -46.81250 & 2.05 & 0.76 & 6.6 & - & $0-0$ & - & - \\
\hline 54637 & ESO 328-41 & 15.30663 & -38.50690 & 1.40 & 0.69 & 3.1 & $S$ & $5-7$ & $9-11$ & 0.17 \\
\hline 56077 & IC 4555 & 15.80448 & -78.17830 & 1.30 & 0.64 & 5.9 & - & $0-0$ & - & - \\
\hline
\end{tabular}


Table 1. continued.

\begin{tabular}{|c|c|c|c|c|c|c|c|c|c|c|}
\hline pgc & galaxy-name & al2000 & de2000 & $\operatorname{logd} 25$ & $\operatorname{logr} 25$ & $t$ & $N / S$ & $(w a) \mathrm{E}-\mathrm{W}\left(^{\circ}\right)$ & $\beta\left({ }^{\circ}\right)$ & $\alpha_{\mathrm{s}}$ \\
\hline 57582 & UGC 10288 & 16.24027 & -0.20780 & 1.68 & 0.94 & 5.3 & - & $0-0$ & - & - \\
\hline 57876 & IC 4595 & 16.34586 & -70.14160 & 1.49 & 0.75 & 5.0 & - & $0-0$ & - & - \\
\hline 58742 & ESO 137-38 & 16.68135 & -60.39340 & 1.48 & 0.60 & 4.4 & ? & $?$ & - & - \\
\hline 59635 & ESO 138-14 & 17.11666 & -62.08300 & 1.57 & 0.80 & 6.7 & - & $0-0$ & - & - \\
\hline 60216 & ESO 138-24 & 17.40184 & -59.38210 & 1.30 & 0.65 & 4.9 & $S$ & 9-9 & $17-17$ & 0 \\
\hline 60595 & IC 4656 & 17.62894 & -63.72950 & 1.39 & 0.61 & 5.0 & - & $0-0$ & - & - \\
\hline 60772 & ESO 139-21 & 17.73619 & -60.97850 & 1.32 & 0.60 & 3.0 & - & $0-0$ & - & - \\
\hline 62024 & IC 4717 & 18.55492 & -57.97400 & 1.22 & 0.61 & 3.0 & - & $0-0$ & - & - \\
\hline 62529 & ESO 281-33 & 18.88273 & -42.53750 & 1.24 & 0.61 & 3.0 & $?$ & $?$ & - & - \\
\hline 62706 & IC 4810 & 19.04974 & -56.15860 & 1.57 & 0.91 & 6.6 & - & $0-0$ & - & - \\
\hline 62722 & NGC 6722 & 19.06100 & -64.89480 & 1.46 & 0.67 & 2.9 & $S$ & $5-5$ & $8-8$ & 0 \\
\hline 62782 & IC 4819 & 19.11826 & -59.46550 & 1.47 & 0.73 & 6.0 & $S$ & $5-2$ & $8-$ & 0.4 \\
\hline 62816 & ESO 231-23 & 19.14551 & -51.04600 & 1.25 & 0.67 & 3.0 & $U$ & $6-6$ & $11-14$ & 0 \\
\hline 62922 & IC 4827 & 19.22261 & -60.86010 & 1.47 & 0.68 & 2.0 & $L N$ & $4-0$ & $8-0$ & 1 \\
\hline 62938 & IC 4832 & 19.23426 & -56.60900 & 1.37 & 0.64 & 1.3 & $N$ & $9-9$ & $17-17$ & 0 \\
\hline 62964 & IC 4837A & 19.25435 & -54.13250 & 1.62 & 0.73 & 3.1 & $N$ & $7-7$ & $15-15$ & 0 \\
\hline 63161 & ESO 184-63 & 19.39455 & -55.06580 & 1.36 & 0.72 & 2.9 & $N$ & $7-6$ & $21-22$ & 0.08 \\
\hline 63297 & ESO 184-74 & 19.50854 & -57.28420 & 1.25 & 0.70 & 2.9 & $?$ & b & - & - \\
\hline 63395 & IC 4872 & 19.59511 & -57.51840 & 1.51 & 0.76 & 6.7 & $S$ & $2-3$ & -11 & 0.2 \\
\hline 63509 & ESO 142-30 & 19.67763 & -60.04800 & 1.26 & 0.67 & 4.9 & $?$ & $\mathrm{~b}$ & - & - \\
\hline 63577 & IC 4885 & 19.73113 & -60.65150 & 1.29 & 0.64 & 4.9 & $S$ & $7-7$ & $11-11$ & 0 \\
\hline 64180 & ESO 105-26 & 20.15782 & -66.21610 & 1.18 & 0.64 & 4.2 & $?$ & $?$ & - & - \\
\hline 64240 & NGC 6875A & 20.19888 & -46.14380 & 1.47 & 0.70 & 4.2 & $?$ & b & - & - \\
\hline 64597 & IC 4992 & 20.39088 & -71.56520 & 1.34 & 0.88 & 5.1 & $S$ & $3-3$ & $8-8$ & 0 \\
\hline 65665 & IC 5054 & 20.89587 & -71.02410 & 1.32 & 0.62 & 1.1 & $?$ & $?$ & - & - \\
\hline 65794 & ESO 286-18 & 20.96403 & -43.37390 & 1.42 & 0.79 & 3.8 & $U$ & $6-2$ & $9-3$ & 0.5 \\
\hline 65915 & IC 5071 & 21.02221 & -72.64490 & 1.53 & 0.67 & 4.8 & $N$ & $6-6$ & $39-23$ & 0 \\
\hline 66064 & ESO 235-53 & 21.08624 & -47.78900 & 1.39 & 0.67 & 3.0 & $N$ & $9-9$ & $22-22$ & 0 \\
\hline 66101 & ESO 235-57 & 21.10602 & -48.16920 & 1.37 & 0.69 & 3.9 & - & $0-0$ & - & - \\
\hline 66530 & IC 5096 & 21.30611 & -63.76130 & 1.50 & 0.71 & 4.0 & $N$ & $8-4$ & $18-11$ & 0.33 \\
\hline 66545 & ESO 145-4 & 21.31419 & -57.64030 & 1.34 & 0.61 & 5.0 & $?$ & $\mathrm{~b}$ & - & - \\
\hline 66617 & ESO 287-9 & 21.35448 & -46.15240 & 1.25 & 0.73 & 4.3 & - & $0-0$ & - & - \\
\hline 66836 & NGC 7064 & 21.48398 & -52.76610 & 1.52 & 0.76 & 5.3 & - & $0-0$ & - & - \\
\hline 67045 & NGC 7090 & 21.60794 & -54.55740 & 1.89 & 0.77 & 5.1 & - & $0-0$ & - & - \\
\hline 67078 & ESO 287-43 & 21.63656 & -43.93260 & 1.30 & 0.77 & 6.1 & - & $0-0$ & - & - \\
\hline 67158 & ESO 531-22 & 21.67475 & -26.52590 & 1.44 & 1.02 & 4.4 & $S$ & $?-4$ & -11 & - \\
\hline 67782 & ESO 288-25 & 21.98822 & -43.86700 & 1.40 & 0.90 & 4.1 & - & $0-0$ & - & - \\
\hline 67904 & NGC 7184 & 22.04401 & -20.81320 & 1.78 & 0.65 & 4.5 & $N$ & $3-3$ & 9-9 & 0 \\
\hline 68223 & IC 5171 & 22.18238 & -46.08210 & 1.38 & 0.61 & 3.8 & $N$ & $6-6$ & $11-11$ & 0 \\
\hline 68329 & NGC 7232A & 22.22810 & -45.89360 & 1.33 & 0.70 & 2.0 & $N$ & $6-6$ & $14-14$ & 0 \\
\hline 68389 & IC 5176 & 22.24848 & -66.84810 & 1.64 & 0.82 & 4.3 & $N$ & $3-3$ & $6-6$ & 0 \\
\hline 69011 & IC 5224 & 22.50824 & -45.99330 & 1.19 & 0.61 & 2.2 & - & $0-0$ & - & - \\
\hline 69161 & NGC 7307 & 22.56463 & -40.93330 & 1.55 & 0.61 & 5.9 & $N$ & $11-10$ & $31-22$ & 0.05 \\
\hline 69539 & NGC 7361 & 22.70498 & -30.05800 & 1.60 & 0.65 & 4.6 & $S$ & $5-5$ & $14-14$ & 0 \\
\hline 69620 & IC 5244 & 22.73710 & -64.04230 & 1.46 & 0.73 & 3.0 & $N$ & $3-2$ & $8-4$ & 0.2 \\
\hline 69661 & NGC 7368 & 22.75876 & -39.34150 & 1.48 & 0.75 & 3.1 & $N$ & $9-6$ & $18-14$ & 0.2 \\
\hline 69707 & IC 5249 & 22.78511 & -64.83150 & 1.59 & 1.07 & 6.8 & - & $0-0$ & - & - \\
\hline 69967 & NGC 7400 & 22.90582 & -45.34670 & 1.41 & 0.63 & 4.0 & - & $0-0$ & - & - \\
\hline
\end{tabular}


Table 1. continued.

\begin{tabular}{clccccccccc}
\hline \hline pgc & galaxy-name & al2000 & de2000 & logd25 & logr25 & $t$ & $N / S$ & $($ wa $)$ E-W $\left(^{\circ}\right)$ & $\beta\left(^{\circ}\right)$ & $\alpha_{\mathrm{s}}$ \\
\hline 70025 & NGC 7416 & 22.92829 & -5.49650 & 1.50 & 0.68 & 3.0 & $S$ & $4-4$ & $9-9$ & 0 \\
70070 & IC 5269B & 22.94356 & -36.24970 & 1.58 & 0.72 & 5.6 & - & $0-0$ & - & - \\
70081 & IC 5264 & 22.94796 & -36.55430 & 1.39 & 0.72 & 2.4 & $N$ & $5-5$ & $16-16$ & 0 \\
70084 & MCG-2-58-11 & 22.94754 & -8.96760 & 1.31 & 0.67 & 4.7 & $S$ & $7-4$ & $16-8$ & 0.27 \\
& & & & & & & & & & \\
70142 & IC 5266 & 22.97244 & -65.12970 & 1.24 & 0.61 & 3.1 & - & $0-0$ & - & - \\
70324 & NGC 7462 & 23.04623 & -40.83400 & 1.62 & 0.71 & 4.1 & - & $0-0$ & - & - \\
71309 & ESO 291-24 & 23.39472 & -42.40210 & 1.22 & 0.61 & 5.0 & $?$ & $?$ & - & - \\
71800 & IC 5333 & 23.58140 & -65.39590 & 1.24 & 0.71 & 3.4 & $L N$ & $7-0$ & $22-$ & 1 \\
71948 & ESO 240-11 & 23.63039 & -47.72630 & 1.74 & 0.92 & 4.8 & - & $0-0$ & - & - \\
72178 & ESO 292-14 & 23.70990 & -44.90460 & 1.43 & 0.88 & 6.5 & $N$ & $2-5$ & $6-11$ & 0.43 \\
\hline
\end{tabular}

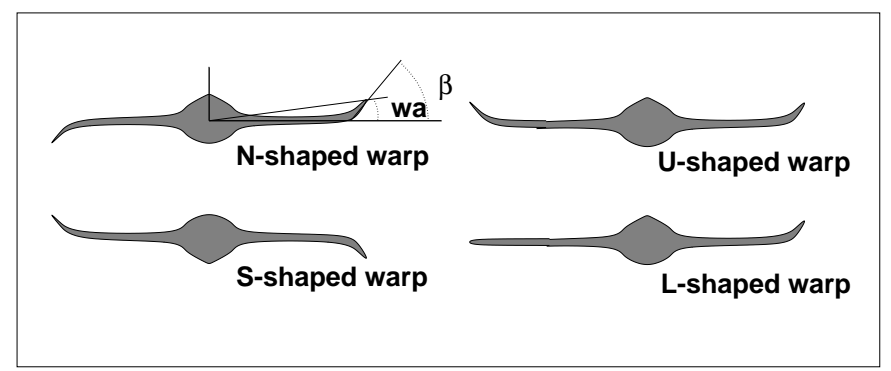

Fig. 1. Definitions of angles and types of warps.

east. $W$, the side of the galaxy closer to the west. In this column, $\mathrm{c}$ indicates the presence of noticeable corrugations, and b means that the observed apparent warps could actually be arms. These are not included as true warps.

The angle $\beta$ in Col. 10 is the angle between the outermost detected point and the point where the warp starts (see Fig. 1).

Finally, Col. 11 gives $\alpha_{\mathrm{s}}$, the degree of asymmetry, defined as

$\alpha_{\mathrm{s}}=\frac{|w a(\mathrm{E})-w a(\mathrm{~W})|}{w a(\mathrm{E})+w a(\mathrm{~W})}$.

The catalog for lenticular galaxies is presented independently in Table 2. In this case, we have considered 26 galaxies with the same limits as those used for spiral galaxies. In addition, galaxies with $\log 25>0.57$ were considered. This enlarged the sample by another 12 lenticulars. This sample is complete with the above-mentioned limits.

\section{Basic results}

Figure 2 gives the distribution of types of warped spiral galaxies, together with the distribution of types for the complete (warped + unwarped) sample. Neither differs significantly from the general distribution of all spirals. The two distributions are so similar, differing only in the size of the sample, that it can be clearly concluded that for spiral galaxies the frequency of warps is completely independent of the type.

The degree of warping is independent of the type, both as defined by the warp angle (wa) (see Fig. 3) and the angle $\beta$ (see Fig. 4). This important property was pointed out by Reshetnikov \& Combes (1999).

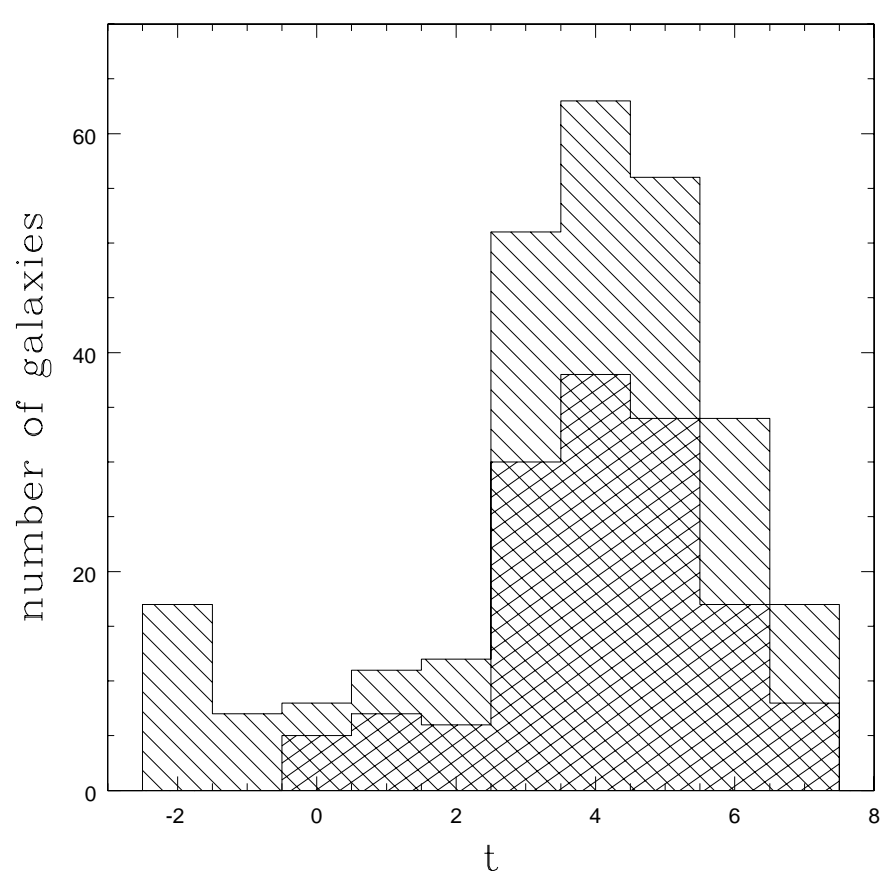

Fig. 2. Distribution of types of warped spiral and lenticular galaxies. Lined shadow area represents all galaxies (warped and unwarped). Squared shadow area represents warped galaxies.

In the case of lenticular galaxies, the result is noticeably different, however. None of the 38 lenticular galaxies in our sample is warped. Warping distorts discs with a similar frequency and amount for $t>0$, but the transition at $t=0$ is very sharp for this feature. This fact could be interpreted in two ways.

Firstly, the lenticular dominant bulges could hamper the formation of warps. There is no obvious theoretical argument favoring this interpretation. On the other hand, the fact that all types of spirals have the same warp frequency and as the size of the bulge is a decreasing function of type, the bulge mass seems to have a small influence on the magnitude of the warp. Therefore, it might be suggested that for a galaxy to be warped it must have large amounts of gas.

Figure 5 shows the relation between $w a$ and $\beta$, which gives some geometrical characteristics of the warp. For moderate warps, there is a clear correlation between $w a$ and $\beta$, as 
Table 2. Sample of lenticular galaxies.

\begin{tabular}{|c|c|c|c|c|c|c|c|}
\hline$\overline{\text { pgc }}$ & galaxy-name & al2000 & 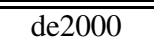 & $\overline{l o g d 25}$ & $\overline{l o g} 25$ & $\bar{t}$ & $\overline{N N / S}$ \\
\hline 5210 & NGC 530 & 1.41159 & -1.58770 & 1.24 & 0.58 & -0.3 & - \\
\hline 5430 & NGC 560 & 1.45706 & -1.91310 & 1.30 & 0.57 & -2.4 & - \\
\hline 6117 & NGC 643 & 1.65349 & -75.01100 & 1.22 & 0.60 & -0.2 & - \\
\hline 12662 & ESO 301-9 & 3.38191 & -42.18790 & 1.30 & 0.69 & -1.7 & - \\
\hline 13169 & NGC 1355 & 3.55654 & -4.99880 & 1.20 & 0.61 & -2.1 & - \\
\hline 13241 & ESO 548-47 & 3.57875 & -19.02900 & 1.40 & 0.60 & -0.8 & - \\
\hline 13277 & IC 335 & 3.59187 & -34.44660 & 1.37 & 0.58 & -1.6 & - \\
\hline 14495 & NGC 1529 & 4.12195 & -62.89900 & 1.10 & 0.58 & -2.2 & - \\
\hline 15388 & IC 2085 & 4.52344 & -54.41690 & 1.35 & 0.64 & -1.2 & - \\
\hline 19811 & NGC 2310 & 6.89821 & -40.86220 & 1.62 & 0.74 & -1.9 & - \\
\hline 24195 & ESO 562-23 & 8.60971 & -20.47010 & 1.36 & 0.61 & -0.9 & - \\
\hline 24966 & ESO 371-26 & 8.90906 & -32.93740 & 1.49 & 0.68 & -1.5 & - \\
\hline 25202 & ESO $90-12$ & 8.97290 & -66.72830 & 1.34 & 0.62 & -1.8 & - \\
\hline 25943 & ESO 433-8 & 9.20360 & -30.91120 & 1.32 & 0.66 & -1.9 & - \\
\hline 30177 & NGC 3203 & 10.32623 & -26.69820 & 1.45 & 0.65 & -1.3 & - \\
\hline 30792 & NGC 3250D & 10.46610 & -39.81490 & 1.24 & 0.71 & -1.9 & - \\
\hline 30938 & IC 2584 & 10.49771 & -34.91160 & 1.16 & 0.57 & -2.0 & - \\
\hline 31369 & MCG-2-27-9 & 10.59093 & -14.12990 & 1.32 & 0.58 & -1.0 & - \\
\hline 31504 & ESO 437-15 & 10.61611 & -28.17810 & 1.33 & 0.58 & -2.0 & - \\
\hline 36417 & NGC 3831 & 11.72187 & -12.87700 & 1.38 & 0.60 & -0.8 & - \\
\hline 37326 & NGC 3957 & 11.90028 & -19.56820 & 1.49 & 0.64 & -1.0 & - \\
\hline 42486 & NGC 4603C & 12.67865 & -40.76350 & 1.24 & 0.59 & -2.0 & - \\
\hline 43929 & NGC 4784 & 12.91030 & -10.61300 & 1.19 & 0.60 & -1.8 & - \\
\hline 45650 & MCG-3-34-4 & 13.16221 & -16.60210 & 1.35 & 0.59 & -1.0 & - \\
\hline 46081 & NGC 5038 & 13.25063 & -15.95170 & 1.19 & 0.62 & -1.9 & - \\
\hline 46150 & NGC 5047 & 13.26347 & -16.51940 & 1.43 & 0.71 & -2.0 & - \\
\hline 46166 & NGC 5049 & 13.26648 & -16.39550 & 1.28 & 0.64 & -2.0 & - \\
\hline 46525 & NGC 5084 & 13.33799 & -21.82700 & 2.03 & 0.60 & -1.8 & - \\
\hline 49006 & ESO 445-42 & 13.81359 & -31.15510 & 1.14 & 0.74 & -0.4 & - \\
\hline 49300 & ESO 445-65 & 13.87963 & -29.92950 & 1.18 & 0.62 & -2.2 & - \\
\hline 49840 & ESO 384-26 & 14.00407 & -34.03760 & 1.19 & 0.57 & -1.9 & - \\
\hline 50242 & IC 4333 & 14.08889 & -84.27290 & 1.20 & 0.62 & -1.8 & - \\
\hline 62692 & NGC 6725 & 19.03230 & -53.86470 & 1.36 & 0.65 & -2.0 & - \\
\hline 63039 & ESO 184-53 & 19.30533 & -53.47730 & 1.10 & 0.60 & -1.8 & - \\
\hline 63049 & NGC 6771 & 19.31105 & -60.54560 & 1.37 & 0.64 & -1.0 & - \\
\hline 65055 & ESO 234-53 & 20.60682 & -49.25780 & 1.28 & 0.59 & -2.0 & - \\
\hline 66908 & ESO 47-34 & 21.52875 & -76.48040 & 1.21 & 0.66 & -2.0 & - \\
\hline 69638 & NGC 7359 & 22.74651 & -23.68700 & 1.35 & 0.58 & -1.8 & - \\
\hline
\end{tabular}

expected. However, for very large values of $\beta$, wa remains constant. Actually, a threshold value for wa seems to exist at around $14^{\circ}$. The farther away the warp starts, the steeper it rises. Theoretical work should pay attention to this fact.

Asymmetry seems to be unrelated to the morphological type. Figure 6 shows that no large asymmetric warps are found in early types, but as the frequency of these early types is much lower, this result cannot be considered significant.
The frequency of warps is summarized in the following table:

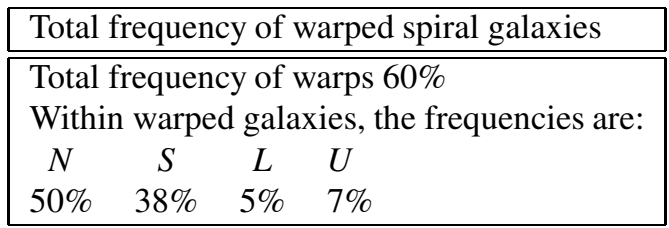




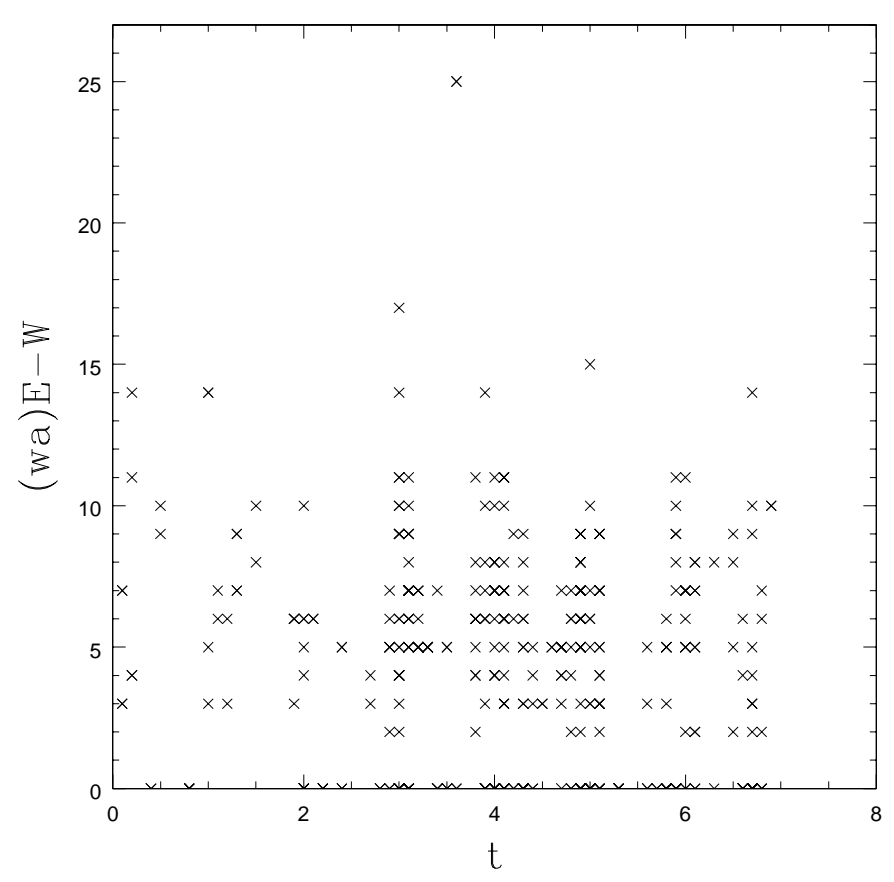

Fig. 3. Warp angle versus type.

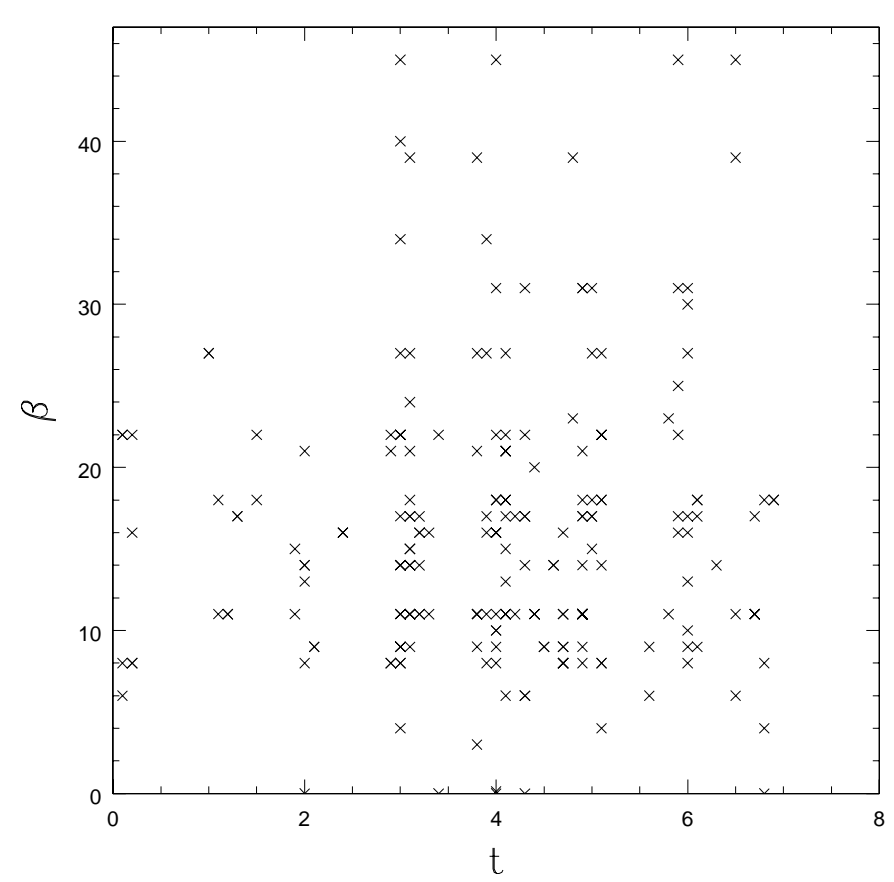

Fig. 4. Angle $\beta$ versus type.

The $N$ and $S$ frequencies are similar, as expected, as these characteristics depend on the observer rather than on the galaxy. The difference, $50 \%$ and $38 \%$, is not significant (it is within the statistical errors). The frequency of $U$ warps is a parameter of theoretical importance, however, the different scenarios predicting different values. As stated by CastroRodríguez et al. (2002) an $L$ warp, or even any asymmetric warp can be interpreted as a $(N+U)$ or as a $(S+U)$ warp.

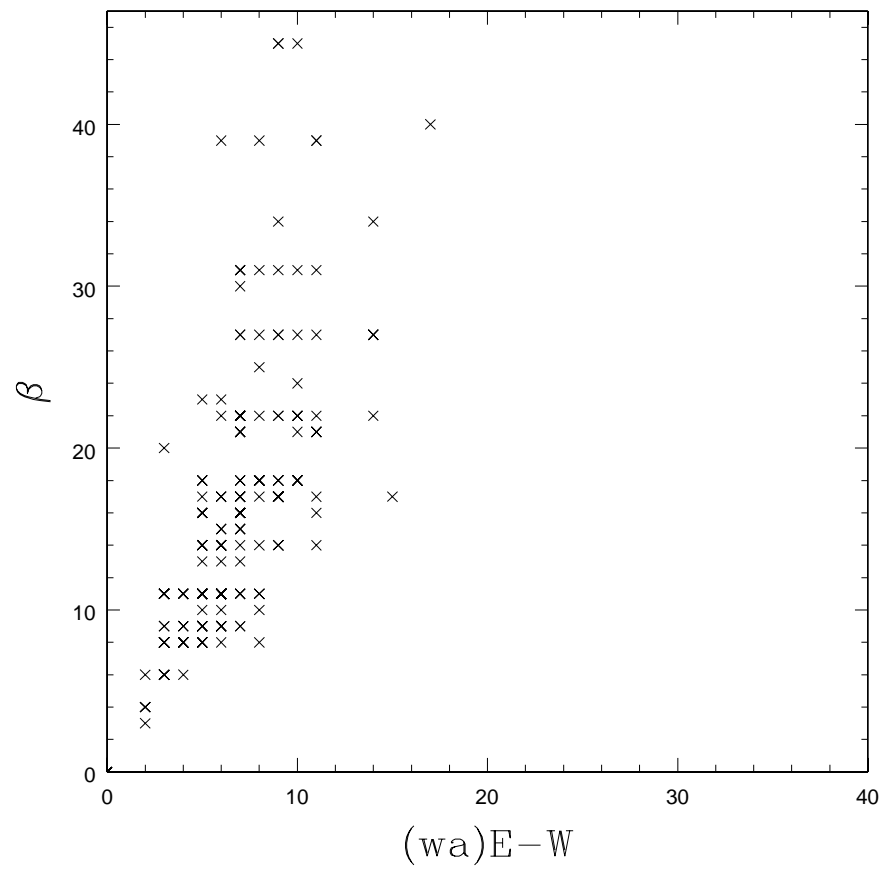

Fig. 5. Relation between $w a$ and $\beta$.

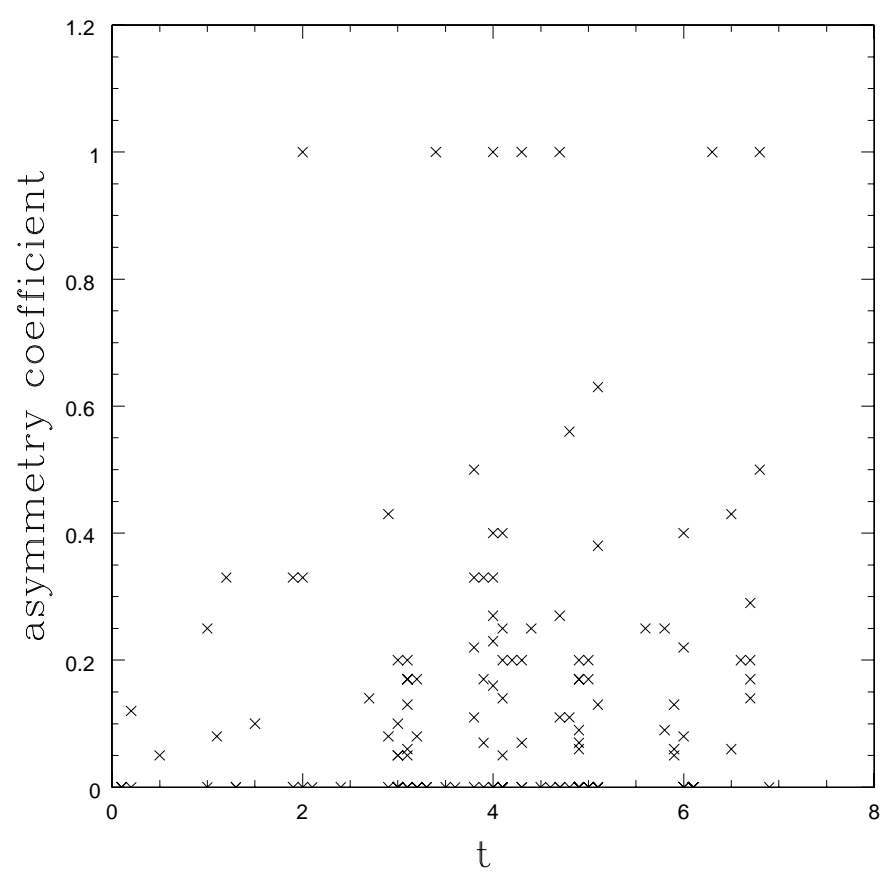

Fig. 6. Asymmetry coefficient versus type.

\section{Conclusions}

1. This catalog contains a large sample of warped galaxies (150), covering the complete southern hemisphere. We present the whole sample of 250 spirals and 26 lenticulars, with limits $\log 25>0.60, B_{\mathrm{t}}<14.5, \delta<0^{\circ},-2.5<t<7$. It is especially suitable for statistical analysis and, indeed, has already been used to study the relation between intrinsic parameters and warps by Castro-Rodríguez et al. (2002). The catalog may obviously be used to choose which galaxies are to be observed in detail. 
2. There is the unavoidable problem of the existence of other contaminant effects that could present the appearance of warps. Reshetnikov \& Combes (1998) estimated that about $20 \%$ of the features assumed to be warps could actually be spiral arms. This value could be applied here. This effect introduces small errors into our calculated frequencies, which should be taken into account in statistical studies.

3. We confirm that warps appear to be a universal feature in spiral galaxies. The frequency of warps is very high (60\%), and because of the difficulty (or impossibility) of detecting warps with the line of nodes in the plane of sky, it is concluded that most (if not all) galaxies are warped. Bosma (1981), Sánchez-Saavedra et al. (1990) and Reshetnikov \& Combes (1999) previously reached this conclusion.

4. We also confirm, by means of a larger amount of data, the finding by Reshetnikov \& Combes (1999) that warps are equally present in all types of spirals. The distribution of warps for the different types of spirals coincides with the distribution of galaxies with type. The maximum observed warp angle either from the center $(w a)$ or from the starting radius of the warps $(\beta)$ has no relation with the type of spiral. We have observed that very large values of $\beta$ do not correspond to large warp angles of $w a$. Shorter warps are steeper.

5. We found no warped lenticular galaxies at all among the 26 galaxies within our limits, with $\log 25>0.60$. We enlarged the sample to reach logr $25>0.57$ and again, none of the 38 lenticulars was warped. There is a sudden transition at $t=0$. All spirals are warped; no lenticular is warped. The main difference between spirals and lenticulars is probably that the former are gas rich and the latter gas poor. Gas seems to be a necessary ingredient in the warp mechanism. Models based on gravitation alone would have serious difficulties in explaining this.

Acknowledgements. We acknowledge the use of the LEDA database (http://leda.univ-lyon1.fr) and the Digitized Sky Survey (DSS) of NASA's SkyView facility (http://skyview.gsfc.nasa.gov) located at NASA Goddard Space Flight Center.

\section{References}

Battaner, E., Florido, E., \& Sánchez-Saavedra, M. L. 1990, A\&A, 236,1

Battaner, E., Garrido, J. L., Sánchez-Saavedra, M. L., \& Florido, E. 1991, A\&A, 251, 402

Binney, J. J. 1992, ARA\&A, 30, 51

Bosma, A. 1981, AJ, 86, 1791

Castro-Rodríguez, N., López-Corredoira, M., Sánchez-Saavedra, M. L., \& Battaner, E. 2002, A\&A, 391, 519

Dubinski, J., \& Kuijken, K. 1995, ApJ, 442, 492

Florido, E., Prieto, M., Battaner, E., Mediavilla, E., \& Sánchez-Saavedra, M. L. 1991, A\&A, 242, 301

García-Ruíz, I. 2001, Ph.D. Thesis, Groningen University

García-Ruíz, I., Kuijken, K., \& Dubinski, J. 2000, MNRAS, submitted, preprint [astro-ph/0002057]

de Grijs, R. 1997, Ph.D. Thesis, Groningen University

Jiménez-Vicente, J. 1998, Ph.D. Thesis, Granada University

Jiménez-Vicente, J., Porcel, C., Sánchez-Saavedra, M. L., \& Battaner, E. 1998, Ap\&SS, 253, 225

Kahn, F. D., \& Woltjer, L. 1959, AJ, 130, 705

Kuijken, K., \& García-Ruíz, I. 2001, Galaxy Disks and Disk Galaxies, ed. J. G. Funes, S. J., \& E. M. Corsini, ASP Conf. Ser., 230, 401

López-Corredoira, M., Betancort, J. A., \& Beckman, J. 2002, A\&A, to be published

Ostriker, E. C., \& Binney, J. J. 1989, MNRAS, 237, 785

Reshetnikov, V., \& Combes, F. 1998, A\&A, 337, 9

Reshetnikov, V., \& Combes, F. 1999, A\&A, 138, 101R

Reshetnikov, V., Battaner, E., Combes, F., \& Jiménez-Vicente, J. 2002, A\&A, 382, 513

Sánchez-Saavedra, M. L., Battaner, E., \& Florido, E. 1990, MNRAS, 246, 458

Sancisi, R. 1976, A\&A, 53, 159

Schwarzkopf, U., \& Dettmar, R. J. 2001, A\&A, 373, 402

Sparke, L., \& Casertano, S. 1988, MNRAS, 234, 873

Weinberg, M. D. 1998, MNRAS, 299, 499 